\title{
Tratamiento periprocedimiento en pacientes con fibrilación auricular que reciben anticoagulantes
}

\section{Periprocedural therapy in patients with atrial fibrillation receiving oral anticoagulation therapy}

\author{
Boris Vladimir Astudillo-Ramírez
}

Servicio de Electrofisiología, Clínica del Rosario, Medellín, Colombia

Recibido el 3 de octubre de 2016; aceptado el 6 de octubre de 2016

Disponible en Internet el 4 de noviembre de 2016

\section{Introducción}

La fibrilación auricular es la arritmia cardiaca más frecuente en el mundo; el 1\% de la población menor de 65 años la padece y llega a estar presente hasta en el $6 \%$ de personas mayores de 80 años. Es un factor independiente en cuanto a morbilidad y mortalidad ${ }^{1,2}$, que aumenta la probabilidad de eventos cerebro-vasculares isquémicos hasta 5,6 veces, y en el que por tanto la anticoagulación se considera fundamental para su tratamiento. Tradicionalmente, el principal anticoagulante ha sido la warfarina (antagonista de la vitamina $\mathrm{K}^{3}$ y más recientemente los inhibidores de la trombina (dabigatrán) ${ }^{4}$ o los inhibidores orales del factor Xa (rivaroxabán, apixabán y edoxabán $)^{5-7}$.

Se estima que cada año, uno de cada seis pacientes que reciben anticoagulación oral con warfarina son intervenidos en procedimientos quirúrgicos o invasivos electivos que requieren la suspensión temporal del medicamento para disminuir el riesgo de sangrado ${ }^{8}$, aumentándose así la probabilidad de fenómenos trombóticos, de ahí que se deba buscar un equilibrio entre el riesgo de embolia (con el uso de escalas como $\mathrm{CHADS}_{2}$ y $\mathrm{CHA}_{2} \mathrm{DS}_{2}$. VASC) y el de sangrado, con el índice de HAS-BLED ${ }^{9,10}$ (tablas 1 y 2 ).

Correo electrónico: vladiastu@gmail.com

\section{Evaluación del riesgo de tromboembolia perioperatoria}

En pacientes con fibrilación auricular, el riesgo de embolia sistémica y eventos cerebro-vasculares isquémicos se evalúa mediante la escala $\mathrm{CHADS}_{2}$, que asigna un punto por cada uno de los siguientes valores: falla cardiaca congestiva, hipertensión arterial, edad superior a 75 años y diabetes, y dos puntos por historia de eventos cerebro-vasculares. El valor de esta escala de riesgo está directamente relacionado con la incidencia de eventos embólicos en pacientes sin anticoagulación ${ }^{11}$.

De otro lado, la escala de $\mathrm{CHA}_{2} \mathrm{DS}_{2}$-VASC, incorporada posteriormente, es una versión modificada de la $\mathrm{CHADS}_{2}$, que incluye enfermedad vascular (infarto agudo de miocardio, placas aórticas o enfermedad vascular periférica), edad entre 65 y 74 años y sexo femenino. Si se adiciona al menos un valor a los factores de riesgo tomados en cuenta en la $\mathrm{CHA}_{2} \mathrm{DS}_{2}$-VASC a los pacientes con $\mathrm{CHADS}_{2}$ de 0 , estos serían candidatos a terapia antitrombótica $^{12,13}$.

Existen otros factores de riesgo evidenciados en el ecocardiograma para eventos trombóticos, como dilatación auricular con contraste espontáneo, disminución de la velocidad doppler en la orejuela izquierda y trombos en la orejuela $^{14}$. 
Tabla 1 Índices de $\mathrm{CHA}_{2} \mathrm{DS}_{2}$-VASC Y HAS-BLED

\begin{tabular}{|c|c|c|c|}
\hline $\mathrm{CHA}_{2} \mathrm{DS}_{2}$-VASC & & HAS-BLED & \\
\hline Hipertensión & 1 & Hipertensión & 1 \\
\hline Evento cerebro-vascular & 2 & $\begin{array}{l}\text { Evento } \\
\text { cerebro-vascular }\end{array}$ & 1 \\
\hline Edad 65 a 74 años & 1 & $\begin{array}{l}\text { Edad mayor } \\
\text { de } 65 \text { años }\end{array}$ & 1 \\
\hline Edad 75 años & 2 & Falla renal & $1 \circ 2$ \\
\hline Mujer & 1 & INR lábil & 1 \\
\hline Enfermedad vascular & 1 & Sangrado & 1 \\
\hline Falla cardiaca & 1 & $\begin{array}{l}\text { Abuso de alcohol } \\
\text { o drogas }\end{array}$ & $1 \circ 2$ \\
\hline Diabetes mellitus & 1 & Puntaje total & 9 \\
\hline
\end{tabular}

\section{Evaluación del riesgo de sangrado perioperatorio}

Esta es anual en pacientes con fibrilación auricular y se basa en la escala clínica HAS-BLED, desarrollada en un seguimiento de eventos de sangrado mayor (hemorragia intracraneal, requerimiento de hospitalización, caída de la hemoglobina $2 \mathrm{~g} / \mathrm{dl}$ y/o transfusión) a 3.978 pacientes que recibieron warfarina; en este seguimiento se identificaron como factores de riesgo: hipertensión arterial (presión arterial sistólica mayor de $160 \mathrm{~mm} \mathrm{Hg}$ ), historia o predisposición a sangrado, INR lábil, edad mayor de 65 años, uso concomitante de antiinflamatorios no esteroides y abuso de drogas o alcohol. El riesgo de sangrado perioperatorio adiciona a la probabilidad de sangrado el riesgo inherente al procedimiento quirúrgico. Se consideran procedimientos de bajo riesgo quirúrgico aquellos con probabilidad de sangrado de $0-2 \%$ y de alto riesgo aquellos de $2-4 \%$ a dos días periprocedimiento ${ }^{15}$.

Tabla 2 Porcentaje de pacientes anticoagulados sometidos a procedimientos

\begin{tabular}{lcc}
\hline Procedimiento & Frecuencia & Porcentaje \% \\
\hline Cirugía oral exodoncia & 1.435 & 14,6 \\
Colonoscopia & 978 & 9,9 \\
Cirugía oftalmológica & 766 & 8,0 \\
Endoscopia superior & 743 & 7,6 \\
Implante de marcapasos & 344 & 3,5 \\
Cistoscopia & 319 & 3,2 \\
Intervención percutánea & 271 & 2,8 \\
$\quad$ coronaria & & \\
Ablación de nodo & 94 & 1,0 \\
$\quad$ aurículo-ventricular & & \\
Aislamiento de venas & 78 & 0,8 \\
$\quad$ pulmonares & & \\
$\quad$ Implante de & 77 & 0,8 \\
$\quad$ cardiodesfibrilador & & \\
\hline
\end{tabular}

Modificado de: Garcia D, Alexander JH, Wallentin L, et al. Management and clinical outcomes in patients treated with apixaban vs. warfarin undergoing procedures. Blood. 2014;124(25): 3692-8.
Tabla 3 Estratificación del riesgo de tromboembolia perioperatoria

\begin{tabular}{ll}
\hline Categoría de riesgo & Fibrilación auricular \\
\hline $\begin{array}{c}\text { Riesgo alto }>10 \% \\
\text { de embolia }\end{array}$ & CHADS $_{2}$ de $5-6$
\end{tabular}

\begin{tabular}{cl} 
& $\begin{array}{l}\text { Accidente isquémico } \\
\text { transitorio o evento } \\
\text { cerebro-vascular }<3 \text { meses } \\
\\
\text { Enfermedad valvular } \\
\text { reumática }\end{array}$ \\
$\begin{array}{c}\text { Riesgo intermedio } \\
4-10 \% \text { embolia }\end{array}$ & CHADS $_{2}$ de $3-4$ \\
Riesgo bajo menor & CHADS $_{2}$ de $0-2$ \\
$>10 \%$ de embolia & \\
\hline
\end{tabular}

Modificada de: Spyropoulos AC, Douketis JD. How i treat anticoagulated patients undergoing an elective procedure or surgery. Blood. 2012;120(15):2954-62.

El tratamiento perioperatorio del paciente con fibrilación auricular, difiere si el paciente es portador de válvula protésica mecánica, considerado como factor independiente para alto riesgo tromboembólico ${ }^{16}$ (tablas 3 y 4).

\section{Tratamiento periprocedimiento de pacientes con fibrilación auricular que reciben antitrombóticos}

En 3.920 pacientes con fibrilación auricular se ha encontrado una correlación del $89 \%$ entre las escalas $\mathrm{CHADS}_{2}$ y HASBLED y de $97 \%$ para $\mathrm{CHA}_{2} \mathrm{DS}_{2}$-VASC/HAS-BLED, y discordancia en el 4,4\% para $\mathrm{CHADS}_{2}$ /HAS-BLED y de 7,7\% para $\mathrm{CHA}_{2} \mathrm{DS}_{2}-$ VASC/HAS-BLED, hecho que confirma la relación entre riesgo tromboembólico y sangrado potencial debido a que ambas escalas comparten factores como edad, hipertensión arterial y antecedentes de eventos cerebro-vasculares ${ }^{17,11}$.

Tabla 4 Riesgo de sangrado por procedimiento

\begin{tabular}{|c|c|}
\hline Alto riesgo $2-4 \%$ & Bajo riesgo $0-2 \%$ \\
\hline $\begin{array}{l}\text { Reemplazo valvular } \\
\text { cardiaco }\end{array}$ & $\begin{array}{l}\text { Endoscopia gastrointestinal } \\
\text { con o sin biopsia }\end{array}$ \\
\hline $\begin{array}{l}\text { Revascularización } \\
\text { quirúrgica } \\
\text { miocárdica }\end{array}$ & $\begin{array}{l}\text { Implante marcapasos, CDI, } \\
\text { estudio electrofisiológico }\end{array}$ \\
\hline $\begin{array}{l}\text { Aneurisma aorta } \\
\text { abdominal }\end{array}$ & $\begin{array}{l}\text { Biopsia cutánea, tiroides, } \\
\text { próstata, nodo }\end{array}$ \\
\hline $\begin{array}{l}\text { Procedimiento neuro- } \\
\text { quirúrgico/urología }\end{array}$ & $\begin{array}{l}\text { Angiografía no coronaria, } \\
\text { retiro de catéter central }\end{array}$ \\
\hline $\begin{array}{l}\text { Cirugía de } \\
\text { cabeza/cuello/cáncer } \\
\text { mama }\end{array}$ & $\begin{array}{l}\text { Cirugía oftalmológica, } \\
\text { catarata/no catarata }\end{array}$ \\
\hline $\begin{array}{l}\text { Extracción dental } \\
\text { múltiple }\end{array}$ & Extracción dental simple \\
\hline $\begin{array}{l}\text { Reemplazo rodi- } \\
\text { lla/laminectomía }\end{array}$ & $\begin{array}{l}\text { Colecistectomía, cirugía } \\
\text { hemorroidal, herniorrafia }\end{array}$ \\
\hline
\end{tabular}




\section{Terapia puente}

Tradicionalmente se ha utilizado la terapia puente en quienes reciben warfarina y han sido sometidos a procedimientos invasivos, recomendación basada en consenso de expertos y estudios observacionales. El BRIDGE es el primer estudio aleatorizado prospectivo que comparó terapia de puente vs. no terapia de puente con la hipótesis de no inferioridad en la incidencia de embolia y disminución en eventos de sangrado para la no terapia de puente. En todos los pacientes se suspendió la warfarina cinco días antes del procedimiento y se aleatorizó en una relación 1:1 a 1.884 pacientes en dos grupos: el primero a terapia de puente $(n=950)$ con administración de dalteparina durante la suspensión, y el segundo a no terapia de puente $(n=934)$, administración de placebo y reinicio del antitrombótico 24 horas después del procedimiento en los pacientes de alto riesgo embólico y 48 horas para los considerados con bajo riesgo. La tasa de tromboembolia arterial en el grupo placebo fue no inferior a la del grupo de terapia de puente $(0,4 \%$ vs. $0,3 \%$; $p=0,01$ para no inferioridad). El sangrado mayor y menor en el grupo placebo fue significativamente más bajo que en el grupo de terapia de puente $(1,3 \%$ vs. $3,2 \%$; $p=0,005 ; 12 \%$ vs. $20,9 \%$; $\mathrm{p}=0,001$; respectivamente) $)^{18}$.

Se excluyeron pacientes con prótesis valvulares cardiacas; en el análisis de las características de los pacientes incluidos en el estudio el promedio de $\mathrm{CHADS}_{2}$ fue de 2,3 incluyendo pocos casos de riesgo tromboembólico sistémico alto (con puntaje de $\mathrm{CHADS}_{2}$ de 5-6). El 89,4\% de los procedimientos a los que fueron sometidos, fueron considerados de bajo riesgo. Este estudio confirma que la terapia sin puente es no inferior para prevenir eventos tromboembólicos y es superior en la reducción de sangrado en pacientes con riesgo intermedio.

Una vez analizado el riesgo de tromboembolia sistémica y de sangrado del paciente con los índices de $\mathrm{CHADS}_{2}$ y HASBLED, las guías de la ACCP establecen cinco grupos; con base en el estudio BRIDGE se plantea una modificación en esta categorización ${ }^{19}$ :

- Categoría A: alto riesgo tromboembólico y alto riesgo de sangrado. Se recomienda considerar la terapia de puente durante el periodo periprocedimiento (clase I, nivel de evidencia C).

- Categoría B: alto riesgo tromboembólico y bajo riesgo de sangrado. Se recomienda terapia de puente en el periprocedimiento (clase ॥, nivel de evidencia C).

- Categoría C: riesgo tromboembólico intermedio y riesgo de sangrado alto. NO usar terapia de puente periprocedimiento (clase I, nivel de evidencia B)

- Categoría D: riesgo tromboembólico intermedio y riesgo de sangrado bajo. NO usar terapia de puente (clase I, nivel de evidencia $B$ ).

- Categoría E: riesgo tromboembólico bajo y riesgo de sangrado bajo. NO terapia de puente (fig. 1).

\section{Esquema perioperatorio con nuevos anticoagulantes}

Los nuevos anticoagulantes (NACO) han adquirido gran importancia; cada vez más pacientes con fibrilación auricular no valvular son tratados con estos medicamentos que actúan como inhibidores directos de la trombina, en el caso de dabigatrán, e inhibidores del factor $x$, en el caso de rivaroxabán, apixabán y edoxabán. En comparación con warfarina, todos han demostrado no inferioridad en la protección del riesgo tromboembólico ${ }^{20}$. La terapia de puente en los pacientes que reciben los NACO no está recomendada debido a la rapidez con la que se alcanza la concentración máxima sérica $\left(C_{\max }\right)$ y su corta vida media, en comparación con la warfarina ${ }^{21-23}$ (tabla 5).

En los estudios de los NACO se ha observado la tasa de suspensión de la medicación para procedimientos invasivos quirúrgicos y no quirúrgicos. Para el dabigatrán, en el estudio RELY a la dosis de $110 \mathrm{mg}$ cada 12 horas, un total de 1.487 pacientes y 1.546 pacientes en el grupo de dabigatrán $150 \mathrm{mg}$ cada 12 horas suspendieron el tratamiento por procedimiento quirúrgico, lo que representa el $25 \%$ de los pacientes de cada brazo. El fármaco fue suspendido 24 horas antes del procedimiento para pacientes con riesgo bajo (depuración de creatinina $>50 \mathrm{ml} / \mathrm{min}$ ) y 3-5 días en pacientes con riesgo alto (depuración de creatinina $<50 \mathrm{ml} / \mathrm{min}$ ); la incidencia de sangrado mayor fue similar en los tres

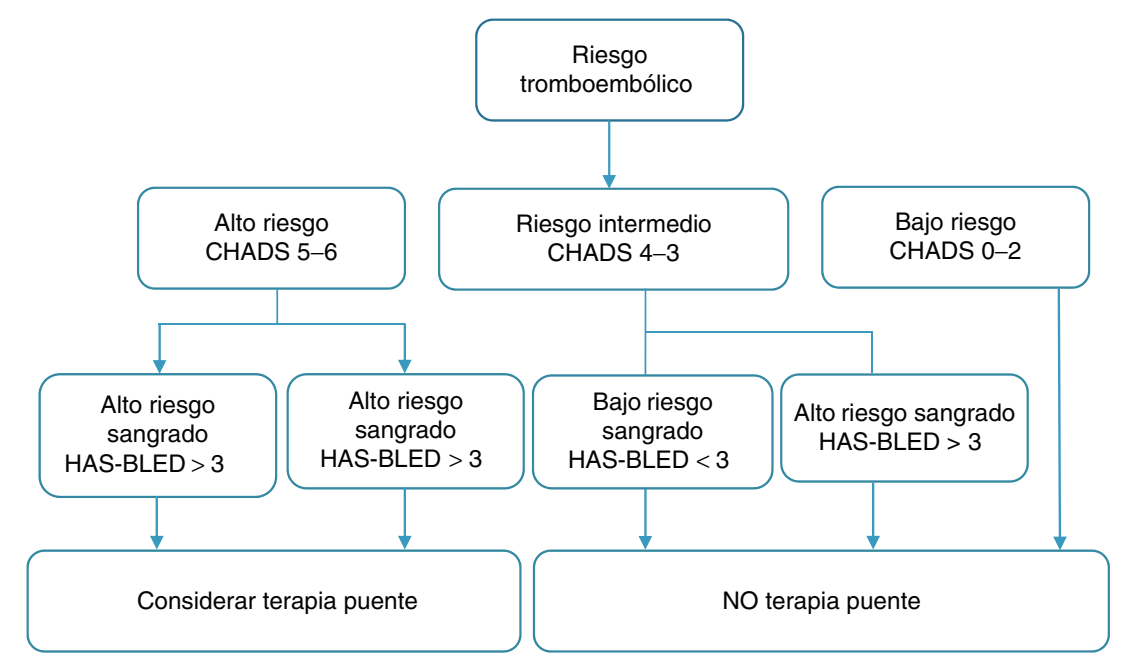

Figura 1 Esquema sugerido para periodo periprocedimiento en pacientes con fibrilación auricular que reciben warfarina. 
Tabla 5 Resumen de los DOAC con antídotos

\begin{tabular}{|c|c|c|c|c|c|c|}
\hline Antitrombótica & Objetivo & Acción & Duración & Excreción & Antídoto & Reversible \\
\hline Warfarina & Vit $\mathrm{K}$ & 24 horas & $72-96 \mathrm{~h}$ & Hepática & FPC\& VK & $12-16 \mathrm{~h}$ \\
\hline HBPM & $\mathrm{Xa}$ & 4 horas & $5-7 \mathrm{~h}$ & $\begin{array}{l}\text { Hepática } 60 \% \\
\text { Renal } 40 \%\end{array}$ & Protamina & Inmediato \\
\hline Rivaroxabán & $\mathrm{Xa}$ & 2 a $4 h$ & $24-48 \mathrm{~h}$ & $\begin{array}{l}\text { Hepática } 66 \% \\
\text { Renal 33\% }\end{array}$ & Andexanet & Completa \\
\hline Apixabán & $\mathrm{Xa}$ & $3-4 h$ & $24-48 \mathrm{~h}$ & $\begin{array}{l}\text { Hepática } 73 \% \\
\text { Renal } 27 \%\end{array}$ & Andexanet & Completa \\
\hline Dabigatrán & Trombina & $0.5-2 \mathrm{~h}$ & $24-96 \mathrm{~h}$ & $\begin{array}{l}\text { Renal } 80 \% \\
\text { Hepática } 20 \%\end{array}$ & Idarucizumab & Completa \\
\hline Edoxabán & $\mathrm{Xa}$ & $1-2 \mathrm{~h}$ & $24-48 \mathrm{~h}$ & Renal 50\% & Andexanet & Completa \\
\hline
\end{tabular}

brazos del estudio (dabigatrán $110 \mathrm{mg}$ 3,8\%, dabiga-

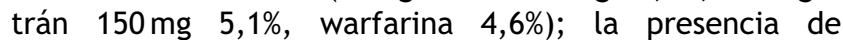
tromboembolia sistémica fue $0,5 \%$ en cada grupo a 30 días del procedimiento ${ }^{24}$.

En el estudio ROCKET, de 14.236 pacientes, 1.309 del grupo rivaroxabán vs. 1.688 del de warfarina, requirieron suspensión del medicamento por intervención quirúrgica o procedimiento invasivo. Los procedimientos más frecuentes fueron los odontológicos, la colonoscopia y la endoscopia digestiva superior; la anticoagulación fue suspendida tres días o más antes del procedimiento en el $90 \%$ de los casos y la terapia puente se usó en 431 pacientes de los dos brazos; estos tenían un $\mathrm{CHADS}_{2}$ de 3,52 vs. 3,40; $\left.\mathrm{p}=0,009\right)$. No se encontraron diferencias significativas en cuanto a la incidencia de tromboembolia sistémica en los pacientes del grupo de rivaroxabán y warfarina $(0,27$ vs. $0,42 \%)$ ni en la tasa de sangrado mayor $(0,99$ vs. $0,97 \%)$ a 30 días del procedimiento.

En el ARISTOTLE, de 18.201 pacientes incluidos en el estudio, 5.439 fueron sometidos a procedimientos invasivos; el $89,8 \%$ fueron no mayores (sin anestesia general) y solo el $2,9 \%$ correspondió a cirugías urgentes. En el $62,1 \%$ del grupo de apixabán y en el 63,0\% del grupo de warfarina se suspendió la medicación y solo el $11,7 \%$ de los pacientes de ambos brazos del grupo recibieron terapia puente. Para aquellos que no interrumpieron la terapia, la tasa de tromboembolia sistémica fue $0,35 \%$ en el grupo de apixabán y $0,57 \%$ en el de warfarina (OR 0,601; 0,322-1.120), mientras que el sangrado mayor fue $1,62 \%$ en el grupo de apixabán y $1,93 \%$ en el de warfarina (OR 0,846; 0,614-1.166). Para los procedimientos en los que se suspendió la terapia, la tasa de tromboembolia sistémica fue 0,31\% para el grupo de apixabán y 0,35\% para el de warfarina, en tanto que el sangrado mayor fue de 1,65\% para apixabán y 1,26\% para warfarina, de lo cual se deduce que no hubo diferencias ni en tromboembolia sistémica ni en sangrado mayor entre los grupos de suspensión de medicación y no suspensión del grupo de apixabán, pero sí más incidencia de sangrado mayor en el grupo de warfarina al suspender la terapia. A partir de estos hallazgos se desprende la seguridad de la no suspensión del apixabán en quienes serán sometidos a procedimientos considerados de bajo riesgo, como implante de marcapasos o aislamiento de venas pulmonares.

De otra parte, Ahmed et al. demostraron en un estudio retrospectivo que la no interrupción de warfarina fue superior a la interrupción programada con o sin terapia de puente en pacientes que serían sometidos a procedimientos de bajo riesgo como implante de marcapasos o desfibriladores, lo cual se reflejó en disminución del hematoma del bolsillo del dispositivo y estancia hospitalaria. Tales hallazgos fueron similares a los del estudio de Tolosana et al. en una población cohorte en la que el 7,8\% del grupo de terapia puente y el $8 \%$ del grupo de no interrupción, desarrollaron hematoma en el sitio de implante del dispositivo $(P=1)$, pero se reportó mayor estancia hospitalaria para el grupo de terapia puente [media de 5 (4-7) vs. 2 (1-4) días; $p<0,001]^{25,26}$.

\section{Grupos especiales}

Los pacientes con válvula protésica y fibrilación auricular fueron excluidos en el estudio BRIDGE y son considerados de alto riesgo tromboembólico. El estudio canadiense PERIOP2, que está en etapa de reclutamiento de pacientes, planea incluir aquellos con prótesis valvulares para evaluar la terapia de puente; hasta entonces la evidencia se basará en consenso de expertos. En caso de riesgo tromboembólico alto y riesgo de sangrado bajo se recomienda la terapia puente; por el contrario, si el riesgo de sangrado es alto y el procedimiento es de bajo riesgo, una alternativa es la no interrupción de warfarina. Si el procedimiento es de alto riesgo se considera la terapia puente, conducta que será desvirtuada o confirmada por el estudio PERIOP2. Los pacientes multivalvulares seguirán siendo una incógnita debido a que fueron excluidos debido a su muy alto riesgo.

\section{Conclusión}

Cerca del 15 al 20\% de los pacientes que reciben anticoagulación requieren interrupción de su tratamiento por procedimientos invasivos o quirúrgicos; en su mayoría, estos procedimientos se consideran de bajo riesgo. Los procedimientos urgentes ocupan menos del $3 \%$ en las estadísticas, con una proporción de sangrado periprocedimiento de hasta seis veces mayor que en procedimientos electivos. La terapia puente queda relegada a pacientes de alto riesgo tromboembólico con un nivel de evidencia $C$ en espera de estudios prospectivos. En pacientes con riesgo tromboembólico intermedio y bajo, no se recomienda la terapia puente. Para los pacientes tratados con nuevos anticoagulantes no se recomienda terapia puente debido a su vida media corta y su rápida concentración máxima sérica $\left(C_{\max }\right)$. En el caso de dabigatrán se suspende la medicación 24-48 horas antes 
si el riesgo de sangrado es bajo y 72-96 horas antes si es alto o la depuración de creatinina es menor a $50 \mathrm{ml} / \mathrm{min}$; en el caso de rivaroxabán se recomienda suspender antes de las 24 horas, en tanto que en el caso del apixabán si se va a suspender la medicación, se recomienda hacerlo antes de 24 horas. En los pacientes de alto riesgo embólico y bajo riesgo de sangrado que irán a procedimientos de bajo riesgo, la terapia con apixabán se podría continuar durante el periodo periprocedimiento.

\section{Conflicto de intereses}

Los autores declaran no tener ningún conflicto de intereses.

\section{Bibliografía}

1. Kannel WB, Abbott RD, Savage DD, McNamara PM. Epidemiologic features of chronic atrial fibrillation: the Framingham study. $\mathrm{N}$ Engl J Med. 1982;306:1018-22.

2. Wolf PA, Abbott RD, Kannel WB. Atrial fibrillation as an independent risk factor for stroke: the Framingham Study. Stroke. 1991;22:983-8.

3. January CT, Wann L, Alpert JS, et al. 2014 AHA/ACC/HRS guideline for the management of patients with atrial fibrillation a report of the American College of Cardiology/American Heart Association Task Force on Practice guidelines and the Heart Rhythm Society. Circulation. 2014;130:e199-267.

4. Connolly SJ, Ezekowitz MD, Yusuf S, et al. Dabigatran versus warfarin in patients with atrial fibrillation. N Engl J Med. 2009;361:1139-51.

5. Patel MR, Kenneth W, Mahaffey KW, et al. Rivaroxaban versus warfarin in nonvalvular atrial fibrillation. N Engl J Med. 2011;365:883-91.

6. Granger CB, Alexander JH, McMurray JV, et al. Apixaban versus warfarin in patients with atrial fibrillation. N Engl J Med. 2011;365:981-92.

7. Giugliano RP, Ruff CT, Braunwald E, et al. Edoxaban versus warfarin in patients with atrial fibrillation. N Engl J Med. 2013;369:2093-104.

8. Steinberg BA, Peterson ED, Kim S, et al. Use and outcomes associated with bridging during anticoagulation interruptions in patients with trial fibrillation: findings from the Outcomes Registry for Better Informed Treatment of Atrial Fibrillation (27). Circulation. 2015;131:488-94.

9. Lip GYH, Nieuwlaat R, Pisters R, et al. Refining clinical risk stratification for predicting stroke and thromboembolism in atrial fibrillation using a novel risk factor-based approach: the euro heart survey on atrial fibrillation. Chest. 2010;137:263-72.

10. Pisters R, Lane DA, Nieuwlaat R, et al. A novel user-friendly score (HAS- BLED) to assess 1-year risk of major bleeding in patients with atrial fibrillation: the euro heart survey. Chest. 2010;138:1093-100.

11. Keogh C, Wallace E, Dillon C, et al. Validation of the $\mathrm{CHADS}_{2}$ clinical prediction rule to predict ischaemic stroke. A systematic review and meta-analysis. Thromb Haemost. 2011;106:528-38.

12. Mason PK, Lake DE, DiMarco JP, et al. Impact of the $\mathrm{CHA}_{2} \mathrm{DS}_{2}$ VASC score on anticoagulation recommendations for atrial fibrillation. Am J Med. 2012;125:603-6.

13. Olesen JB, Torp-Pedersen C, Hansen ML, et al. The value of the $\mathrm{CHA}_{2} \mathrm{DS}_{2}$-VASC score for refining stroke risk stratification in patients with atrial fibrillation with a $\mathrm{CHADS}_{2}$ score 0-1: a nationwide cohort study. Thromb Haemost. 2012;107:1172-9.

14. Suzuky T, Yamasaky T, Ogawa S, et al. Echocardiographic predictors of frequency of paroxysmal atrial fibrillation (AF) and its progression to persistent AF in hypertensive patients with paroxysmal AF: results from the Japanese Rhythm Management Trial II for Atrial Fibrillation (J-RHYTHM II Study). Heart Rhythm. 2011;8:1831-6.

15. Claridge SB, Kanaganayagam GS, Kotecha T. Atrial fibrillation guidelines. Don't forget HASBLED score. BMJ. 2011;24:342.

16. Cannegieter SC, Rosendaal FR, Briët E. Thromboembolic and bleeding complications in patients with mechanical heart valve prostheses. Circulation. 1994;89:635-41.

17. Friberg L, Rosenqvist M, Lip GY. Evaluation of risk stratification schemes for schaemic stroke and bleeding in 182678 patients with atrial fibrillation: the Swedish atrial fibrillation cohort study. Eur Heart J. 2012;33:1500-10.

18. Douketis JD, Spyropoulos AC, Kaatz S, et al. Perioperative bridging anticoagulation in patients with atrial fibrillation. N Engl J Med. N Engl J Med. 2015;373:823-33.

19. Douketis JD, Spyropoulos AC, Spencer FA, et al. Perioperative management of antithrombotic therapy: Antithrombotic Therapy and Prevention of Thrombosis, $9^{\text {th }}$, Ed.: American College of Chest Physicians Evidence-Based Clinical Practice Guidelines. Chest. 2012;141 2 Suppl, e326S-50S.

20. Heidbuchel H, Verhamme P, Alings M. EHRA Practical Guide on the use of new oral anticoagulants in patients with non-valvular atrial fibrillation: executive summary. European Heart Journal. Eur Heart J. 2013;34:2094-106.

21. Schulman S, Kearon C, Kakkar AK, et al. Extended use of dabigatran, warfarin, or placebo in venous thromboembolism. N Engl J Med. 2013;368:709-18.

22. Agnelli G, Buller HR, Cohen A, et al. Oral apixaban for the treatment of acute venous thromboembolism. N Engl J Med. 2013;369:799-808.

23. Garcia D, Alexander JH, Wallentin L, et al. Management and clinical outcomes in patients treated with apixaban vs. warfarin undergoing procedures. Blood. 2014;124:3692-8.

24. Rechenmacher SJ, Fang JC. Bridging anticoagulation: primum non nocere. J Am Coll Cardiol. 2015;66:1392-403.

25. Ahmed I, Gertner E, Nelson WB, et al. Continuing warfarin therapy is superior to interrupting warfarin with or without bridging anticoagulation therapy in patients undergoing pacemaker and defibrillator implantation. HRTHM. Elsevier Inc; 2010; 7(6):7459.

26. Tolosana JM, Berne P, Mont L, et al. Preparation for pacemaker or implantable cardiac defibrillator implants in patients with high risk of thrombo-embolic events: oral anticoagulation or bridging with intravenous heparin? A prospective randomized trial. Eur Heart J. 2009;30:1880-4. 